\title{
Natural killer cells, ageing and cancer
}

\author{
Elissaveta Naumova ${ }^{1}$ Graham Pawelec $^{2} \cdot$ Anastasiya Mihaylova ${ }^{1}$
}

Received: 1 September 2015 / Accepted: 25 February 2016 / Published online: 11 March 2016

(C) Springer-Verlag Berlin Heidelberg 2016

\begin{abstract}
Natural killer (NK) cells are key components of innate immunity and substantially contribute to antitumor immune responses. The role of NK cells in immune surveillance is linked to many aspects of NK cell biology, but the age of the animal being studied or the human under treatment is rarely taken into account. The solicited reviews constituting a collection of papers presented here as a "Symposium-in-Writing" on the topic of NK cells, ageing and cancer were inspired by the increasing knowledge of NK cell biology and genetics, and emerging data on their impact in the clinic (disease associations and therapies), together with the realization that older individuals also differ from younger ones regarding innate as well as adaptive immunity.
\end{abstract}

Keywords NK cells $\cdot$ Ageing $\cdot$ Cancer $\cdot$ NK cell therapy

This Editorial forms an introduction to the Symposium-inWriting: "Natural killer cells, ageing and cancer." We thank Prof. Elissaveta Naumova for compiling and co-editing this series of papers and all authors and peer-reviewers for their contributions.

This Editorial refers to the articles available at DOI: $10.1007 /$ s00262-015-1750-0, 10.1007/s00262-016-1806-9, 10.1007/ s00262-016-1803-z, 10.1007/s00262-015-1720-6, 10.1007/ s00262-015-1744-y, 10.1007/s00262-016-1792-y, 10.1007/ s00262-015-1761-x.

Elissaveta Naumova

naumovaej@gmail.com

1 Department of Clinical Immunology and Stem Cell Bank, University Hospital "Alexandrovska", Medical University, 1, Georgi Sofiiski str., 1431 Sofia, Bulgaria

2 Center for Medical Research (ZMF), University of Tübingen Medical School, Tübingen, Germany

\begin{abstract}
Abbreviations
AML Acute myeloid leukemia

HLA Human leukocyte antigen

HSCT Hematopoietic stem cell transplantation

KIR Killer cell immunoglobulin-like receptors

NK Natural killer
\end{abstract}

\section{Introduction}

Natural killer (NK) cells are key components of innate immunity and contribute substantially to anti-tumor immune responses. The role of NK cells in immune surveillance is linked to many aspects of NK cell biology. Despite their discovery more than 40 years ago, new and exciting areas of NK cell function and the molecular mechanisms that govern their reactivity continue to emerge. The "Symposium-in-Writing on NK cells, ageing and cancer" was inspired by the increasing knowledge of NK cell biology and genetics, and emerging data on their impact in the clinic (disease associations and therapies). Although the role of NK cells is clearly established in cancer, we still have much to learn about the molecular basis of their anti-tumor activity. We now know that the number of NK cells generally increases with age, but their functionality on a per-cell basis decreases. As the risk of solid cancer development also increases with age, this raises the question whether the higher incidence of malignancies in older people is related to age-associated alterations of NK cell function. Some of the variables influencing NK cell activity are related to their receptor-ligand interactions, especially those of the two highly polymorphic immune gene systems KIR and HLA. How the genetic background, expression and function of both activating and inhibitory receptors are involved in the regulation of NK cell activities in malignant 
settings is a matter of debate. In addition, the exact link between receptor-ligand genetic profiles, NK cell function and cancer development is still unclear and under intensive investigation. As evidence of NK cell importance in host immunity against cancer increases, the potential clinical application of NK cell-based immunotherapy for malignant diseases is becoming increasingly attractive and more promising.

\section{Outline of the "Symposium-in-Writing on NK cells, ageing and cancer"}

In this "Symposium-in-Writing on NK cells, ageing and cancer," a number of experts have reviewed the data available in the literature, along with their own experience, to consider the biological role of NK cells, the molecular basis of NK cell function and the behavior of NK cells in ageing, cancer and immunotherapy.

Manser and Uhrberg [1] comprehensively consider the impact of age-related changes on the NK cell repertoire and on the increased incidence of malignant disease and mortality in the elderly. NK cell populations go through major changes from newborns to adults characterized by transition from an NKG2A- to a KIR-dominated NK cell repertoire and a mostly cytokine-driven shift in effector function to a more cytotoxic response. Later life mechanisms responsible for the reduced per-cell killing capacity of NK cells (but retained capacity for IFN $\gamma$ and $\mathrm{TNF} \alpha$ production) may be decreased release of perforins, but whether this is generally the case, and if so, why, remains under discussion.

How NK cells may be relevant in hematologic malignancies, some of which are much more common in the elderly than in the young, is considered by Varbanova et al. [2] who present a detailed critical review of the increasing evidence for the relevance of KIR and HLA class I ligand genetic background in the development of these diseases. Summarizing the current data, they conclude that two genetic profiles could be outlined in hemoblastosis which are associated either with higher levels of KIR-mediated inhibition or with a high level of KIR-mediated activation of NK cell function. The inhibitory KIR-ligand combinations may favor the escape of blast cells from NK cell immune surveillance, while the activating KIR genotypes may have opposite effects depending on whether infection is involved in stimulating lymphoproliferation. In addition, these authors discuss their own data showing increased prevalence of inhibitory KIR-ligand profiles in leukemia patients. Finally, they integrate the potential impact of the two highly polymorphic gene systems-KIR and HLA-to represent the association of the control of NK cell reactivity with the promotion of or protection from blood cancers.
Possible relationships between "senescent" and "exhausted" CD8 + T cells and the acquired expression of certain NK receptors and NK-like characteristics are discussed by Kared et al. [3] who summarize their function and possible correlation with clinical prognosis in chronic immune diseases and cancer. The close relationship of CD57 expression with KLRG1 on T cells and with NKG2 on NK cells is discussed, and the differences observed between "senescent" cells and CD57+ "exhausted" T cells consequent to persistent chronic infection are highlighted, as there has been much confusion regarding this nomenclature and the state of these late-stage differentiated cells and their markers. It has been suggested that the CD8+CD57+ $\mathrm{T}$ cell population is functionally heterogeneous and composed of competing (cytotoxic and immunosuppressive, proliferative and terminally differentiated, senescent) subsets; thus, the overall parameter measured depends on the predominance of a particular subset. Hence, the authors propose a model for the acquisition of CD57 during $\mathrm{T}$ and NK cell differentiation of great relevance for the state of immunity in the elderly.

Focusing on a malignancy not especially associated with advanced age, Sanchez-Correa et al. [4] summarize the latest results regarding age-related changes in NK cells and their correlation with age-associated diseases, focusing on acute myeloid leukemia (AML). Based on their extensive experience investigating phenotypes of NK cells in healthy individuals of different ages, as well as the impact of chronic viral infections on NK cells, the authors scrutinize conflicting data from the literature to provide a consensus view. They interpret changes in the phenotype and function of NK cells found in AML patients as paralleling ageassociated alterations or those mostly related to the cancer condition. Finally, they schematically present the complex balance between inhibitory and activating receptor signaling and how activating receptors cooperate to activate NK cell cytotoxicity needed for the development of new therapeutic strategies.

However, successes in clinical trials using NK cellbased immunotherapy for hematologic malignancies are poor so far. To this end, Pietra et al. [5] discuss putative mechanisms by which tumor cells might escape NK cell attack. The influence of the tumor microenvironment on NK cell function in different cancers and on the bone marrow microenvironment for leukemia progression is considered in detail and in the light of the latest studies. Updates related to the use of NK cells in immunotherapy of solid tumors and leukemia suggest that different therapeutic approaches using NK cells boosted by cytokines may be most likely eventually to succeed. Preclinical cancer models and early-stage clinical trials using IL-12 and IL-18 as well as IL-15 as an alternative to the previously commonly used IL-2 for inducing LAK cells do now show some 
promising results. A number of chemotherapeutic drugs may also enhance the expression of TNF-related apoptosisinducing ligand (TRAIL) and NKG2D ligands by tumor cells that consequently become more susceptible to NK cell-mediated cytotoxicity. The use of anti-KIR monoclonal antibodies that disrupt the KIR/HLA class I interaction at the tumor cell surface is a novel attractive therapeutic strategy not only for hematologic malignancies but also for solid tumors. Furthermore, considering the fact that NK cells are the first cellular population regenerating after hematopoietic stem cell transplantation (HSCT), the impact of alloreactive NK cells and the effects of KIR-ligand mismatch in HSCT are well documented. Donor selection criteria additional to the already accepted criteria, including the identification of NK alloreactive cells in potential donors, and genotype analysis aiming to detect KIR profiles associated with more significant activity of NK cells, are under development.

The contribution of Becker et al. [6] takes this discussion further by extensively reviewing the therapeutic potential of NK cells in HSCT and several principles of NK cellbased immunotherapy. New approaches for selection and expansion of primary NK cells for clinical application from different sources including peripheral blood, cord blood, bone marrow, embryonic stem cells, induced pluripotent stem cells as well as NK cell lines/chimeric antigen receptor modification are all being considered.

Cellular therapy as a rapidly developing area opens new horizons in clinical medicine and is a challenge in therapy of cancer. In this context, Suck et al. [7] also review their group's work on the potential for cellular therapy of cancer using the allogeneic natural killer cell line NK-92. The results of preclinical testing of NK-92 in mice indicate that it is well tolerated and lacks toxicity (suitable for clinical application). The efficacy of the cell line as a purging agent and in cGMP-compliant experimental cell cultures has been demonstrated, but phase I/II clinical trials have delivered disappointing results so far. However, genetic engineering of T cells and NK cells to enable them to recognize a specific target antigen and increase their effectiveness is described in this paper, documenting safety in vitro and in vivo in mouse models and possible future application in the clinic.

\section{Conclusions}

Data obtained from the studies reviewed in this series of papers, along with the authors' own experience and expertise, have contributed to a better understanding of the complex nature of NK cells and pointed to the differences also in these innate cells in young and old individuals. This series of papers also highlights controversial data and potential future prospects to resolve these in NK cells, cancer and age interactions. As an example, the age-related transition from an NKG2A- to a more KIR-driven NK cell repertoire may need to be considered, due to increased KIR expression during the first decade with a significant increase in the first 2 years of life. In contrast, neonatal NK cell repertoires are dominated by NKG2A expression. In later life, the question is whether truly immunosenescent NK cells accumulate in the way that CD8 $+\mathrm{T}$ cells do.

We must also note and exercise caution regarding differences between mice and humans, with different constellations of age-related changes in NK cells being observed. A possible reason contributing to this might be the fact that laboratory mice are not exposed to the range of pathogens that humans are. In particular, the relationship between cytomegalovirus (CMV) infection and physiological ageing in humans remains incompletely understood in terms of changes in the cells resulting from infections confounding the purely ageing effects.

The possibility of modulating potentially senescent CD57+ NK cells may be a candidate for an innovative therapeutic strategy (new adoptive immunotherapy, vaccine, etc.). Studies of NK cells have mainly been performed using peripheral blood NK cells in humans. Thus, further studies of NK cells in other immunological organs such as secondary lymphoid organs, liver and bone marrow are needed. These considerations regarding NK cells are essentially the same as for other immune cells, but have not yet been closely approached in the former case. Similarly, thus far unclear issues related to dynamic changes in the number and function of NK cells as targets for future investigations need to be taken into account and are considered by several of the authors of the papers in this series.

While clinical results using NK cells for treatment of leukemia are relatively good, application of NK cells for the treatment of solid tumors has been limited and rather unsuccessful. Recently, the role of immunogenetic factors in predisposition to and protection against hematologic neoplasias and their biological relevance has been intensively discussed. Identification and characterization of fine mechanisms of NK cell function on the one hand and cellular interactions in the tumor microenvironment on the other will facilitate the development of new therapeutic strategies for enhancing the anti-tumor response. Whether these new tools can improve NK cell function and/or increase susceptibility to tumor NK-mediated killing remains to be explored. The collection of papers presented in this "Symposium-in-Writing on NK cells, ageing and cancer" is intended to provide a basis for further contemplation of this important field of research. 


\section{Compliance with ethical standards}

Conflict of interest The authors have no conflict of interest to declare.

\section{References}

1. Manser AR, Uhrberg M (2015) Age-related changes in natural killer cell repertoires: impact on NK cell function and immune surveillance. Cancer Immunol Immunother. doi:10.1007/ s00262-015-1750-0

2. Varbanova V, Naumova E, Mihaylova A (2016) Killer-cell immunoglobulin-like receptor genes and ligands and their role in hematologic malignancies. Cancer Immunol Immunother. doi:10.1007/s00262-016-1806-9

3. Kared H, Martelli S, Ng TP, Pender SL, Larbi A (2016) CD57 in human natural killer cells and T-lymphocytes. Cancer Immunol Immunother. doi:10.1007/s00262-016-1803-z
4. Sanchez-Correa B, Campos C, Pera A, Bergua JM, Arcos MJ, Bañas H, Casado JG, Morgado S, Duran E, Solana R, Tarazona R (2015) Natural killer cell immunosenescence in acute myeloid leukaemia patients: new targets for immunotherapeutic strategies? Cancer Immunol Immunother. doi:10.1007/ s00262-015-1720-6

5. Pietra G, Vitale C, Pende D, Bertaina A, Moretta F, Falco M, Vacca P, Montaldo E, Cantoni C, Mingari MC, Moretta A, Locatelli F, Moretta L (2015) Human natural killer cells: news in the therapy of solid tumors and high-risk leukemias. Cancer Immunol Immunother. doi:10.1007/s00262-015-1744-y

6. Becker PS, Suck G, Nowakowska P, Ullrich E, Seifried E, Bader P, Tonn T, Seidl C (2016) Selection and expansion of natural killer cells for NK cell-based immunotherapy. Cancer Immunol Immunother. doi:10.1007/s00262-016-1792-y

7. Suck G, Odendahl M, Nowakowska P, Seidl C, Wels WS, Klingemann HG, Tonn T (2015) NK-92: an 'off-the-shelf therapeutic' for adoptive natural killer cell-based cancer immunotherapy. Cancer Immunol Immunother. doi:10.1007/s00262-015-1761-x 